\title{
Building Towards Expertise
}

\author{
Marcel Martin ${ }^{1, \text { a }}$, Paul Ouellet ${ }^{2, \mathrm{~b}}$, Zeesham Aslam ${ }^{3, \mathrm{c}}$ \\ ${ }^{1}$ Université de Sherbrooke, Sherbrooke, Quebec, Canada; \\ Dr Everett Chalmers Hospital, Horizon Health Authority, New Brunswick, Canada; \\ ${ }^{2}$ Department of Surgery, Université de Sherbrooke, Sherbrooke, Quebec, Canada; \\ Vitalité Health Authority, North West Zone, New Brunswick; \\ ${ }^{3}$ Dr Everett Chalmers Hospital, Horizon Health Authority, New Brunswick, Canada; \\ Department of Critical Care - Internal Medicine, Dalhousie University, Halifax, Nova Scotia, Canada. \\ a'Marcel.Martin@Usherbrooke.ca, bpo1953@me.com, 'CZeeaslam@me.com
}

Keywords: Continuous medical education, practical knowledge decision-making, simulation laboratory and post-simulation era, neuroscience with modulators, mental practice and artificial intelligence.

\begin{abstract}
To build towards expertise, one has to accept to modify his way of practicing, including: 1) A need to reflect on and about the action and near the action in 'in situ' laboratory, 2) A continuous concern about our competence to practice, (presently medical education is in fact discontinuous with periods of incompetence) 3) Tireless effort to combine metacognition and mental practice in a trans-disciplinary approach, 4) Practice cannot be complete without adding research with neuroscience, understanding neuroplasticity, modulation and artificial intelligence.
\end{abstract}

\section{Introduction}

Continuous education is a mandatory condition of practice 'sine qua non' to maintain at least a competence in 'complex system' like medicine [1,2]. Continuous education should not mean intermittent one with inappropriate activities to learn and retain during these active practicing years. The present system is based on 'one fits all': ATLS, ACLS, FCCS and other similar sessions. Retrospectively, the pre doctoral 'Problem Based Learning' (PBL) approach alone has been an incomplete one mainly used for decision-making and theoretical knowledge training [3]. The practical field of procedural knowledge has been left aside in curriculum like the development of spatial intelligence [4]. Spatial intelligence development has been shown to help in mental representation and therefore must be included in the neuromodulation program.

These reductionist aspects of our curriculum in medicine led to an incomplete metacognition development [5] where procedural knowledge, third space visualisation, anatomy and spatial intelligence were neglected. Mental practices should even replace the present simulation laboratory concept.

Flexner [6] in his time mandates continuity in pre/postdoctoral and continuous education curriculum. This remained only a dream and continuous education has now to be rebuilt again with a complete change in philosophy [7]. In our present work, we insisted on simulation laboratory [8] as an opening to an integration of procedural knowledge, mixed with decision-making, intuition, uncertainties [9, 10] empathy, resilience [11], development of spatial intelligence and creativity. We feel all of the above must be approached in simulation with a global philosophy including technical and non-technical skills and kinetics of decision-making, interdisciplinary approach for both procedural and theoretical knowledge. All those learning elements are included in complex scenarios.

From the world of simulation, the second part of the revolution is the inclusion of neuroscience [12] in cognitive psychology to develop a better metacognition [13] and allow a personalised approach in the development of expertise [14]. The neuroenhancement phenomenon [15], based on neuroplasticity [16] and epigenetics [17] needs to be included for the understanding of neuroplasticity, 
connectomics [18] and neuroimaging [19]. The final aspect of this revolution must be the 'Mental Practice' [20] with the understanding of 'Motor to Mental Gradient' in skill learning [21, 22].

Skill learning should include gradient between cognition, plasticity, epigenetics [23], modification following physical exercise [24] and their influence on learning. All of these elements will finally help in understanding metacognition and contextualisation of knowledge. We are witnessing a post simulation era where mental world and practice with virtual reality would replace the actual simulation laboratory.

One appropriate question is raised. Why such a presentation on cognition at a biotechnology congress? Three main facts transpire.

1- In cognitive psychology, we are utilising more and more 'Technology-Enhanced Education' (TEE).

2- Our learning approach in medicine is partly transferable to 'Science, Technology, Engineering and Mathematics' (STEM) and biotechnology education.

Therefore it is fair to say that in complex systems, learning should more and more be focused on a trans disciplinary approach.

\section{Simulation}

Five main subjects are thoroughly discussed in Simulation laboratory literature:

1- Scenarios with more or less high fidelity,

2- Complex scenarios,

3- Near work space teaching (in situ) with debriefing [25],

4- Teaching in a distributed fashion [26], with or without cognitive task analysis [27]

5- Decomposition of movements [28], consisting of multidisciplinary approach [29] with videocameras [30] for further modeling [31].

For the last twenty years these laboratories have been the revolutionary tools in teaching medicine. Digging on metacognition and cognitive psychology and because of the understanding of the Motor to Mental Gradient in skill learning in sports, mental representation and mental practice started recently to complement the physical practice and to replace it almost completely. Parallel to this approach, neuroscience has helped to understand neuroplasticity, epigenetics and connectomics. Neuroimaging offers a possible follow-up on the road towards expertise.

\section{Mental Practice and Neuroenhancers}

Mental practice and representation with all the other neuroenhancers described recently at least five elements; three of which are environmental (1-3) and two are structural (4-5)

1- Exercise [24]

2- Practice [32]

3- Videogames [33]

4- Biochemical approach with methylene blue or methylphenidate [34]

5- Electro-mechanical stimulation of the brain [35]

All of these mandate a strategic approach [36] and a strict neuroethics [37]. With the recent description of asymptotic [38] curve of expertise, the use of a global strategic approach will contribute to achieve expertise in complex systems. In the coming years, the methodology for efficient learning will change drastically. Result evaluation is mandatory. Recent literature on cognitive psychology is rapidly growing. One example is with the use of music instrument in learning toward neuroplasticity [39], coupled with physical exercise [40] and mental practice with mindfulness meditation [41] to control emotional stress and learning. Among the enhancement literature, Hardy et al. proved the effect of cognitive enhance learning (Videogame Lumosity) in the Grand Index Score being enhanced in speed of processing, short term memory, working memory, problem solving and fluid reasoning assessments [42]. Alam and Leblanc have shown that multiple 
choice scores can be optimized with E-learning sessions by combining mental practice with modeling [43]. Several combinations of enhancement are possible and the operation strategy must be studied in different situations.

\section{Results}

We have reproduced the effect of mental representation coupled with kinesiology and cognitive task analysis for the retention of procedural knowledge in a novice situation (medical student first year) [28]. Realizing more and more the associations of mental imagery and visuo-spatial abilities, we have started very recently to use BCI-MI development establishing a follow-up program with fMRI. Preliminary results should come soon. M.E. Whitcomb in Academic Medicine has recently discussed adequately the need for a major change in the philosophy of continuous education [7].

\section{Summary}

This reflective revolution in education should also include the development of artificial intelligence in a brain-machine interface. However, the application of brain-machine interface mandates a brain control over the machine. Robotics must not replace human brain but must enhance its possibilities. Integration and adaptation must prevail in any learning approach. On the other hand, neuroscience and cognition especially in inhibitory:excitatory (I:E) balance synapses [43] concerning neuroplasticity, should be better understood to optimize the strategy of Targeted Neuroplasticity Training (TNT). By achieving better understanding of cognition and metacognition with neuroimaging, we feel global knowledge will evolve on two fronts : 1- Precision in education with personalized cognition and 2- With combined utilization of neuroscience, artificial intelligence and metacognition evolution will reach for solving the recent asymptote curve of expertise but not without a strict necessity of neuroethics.

\section{References}

[1] Papachristofi O, Jenkins D, Sharples LD. Assessment of learning curves in complex surgical interventions: a consecutive case-series. Trials 17 (2016) 266.

[2] Koh G. Revisiting the 'Essentials of problem based learning'. Medical Education 502016 596-599.

[3] Servant VFC, Schmidt HG. Revisiting 'Foundations of problem-based learning: Some explanatory notes'. Medical Education 50 (2016) 698-701.

[4] Langlois J, Bellemare C, Toulouse J, Wells GA. Spatial abilities and technical skills performance in health care: A systematic review. Medical Education 49 (2015) 1065-1085.

[5] Sternberg RJ. Metacognition, abilities and developing expertise: What makes an expert student? Instructional Science 26 (1998) 127-140.

[6] Arky RA. The family business- To educate. The New England Journal of Medicine 354 (2006) 1922-26.

[7] Whitcomb ME. Transforming Medical Education: Is competency-based medical education the right approach? Academic Medicine 91 (2016) 618-620.

[8] Bond W, Kuhn G, Binstadt E, et al. The use of simulation in the development of individual cognitive expertise in emergency medicine. Academic Emergency Medicine 15 (2008) 1037-1045.

[9] Kepecs A. The uncertainty of it all. Nature Neuro Science 16 (2013) 660-662.

[10] Han PKJ, Klein WMP, Arora N. Varieties of uncertainty in health care: A conceptual taxonomy. Medical Decision Making 31 (2011) 828-838.

[11] Preusche I, Lamm C, Reflections on empathy in medical education: What can we learn from social neurosciences? Advances in Health Science Education 21 (2016) 235-249.

[12] The Royal Society: Neuroscience; Implications for Education and Lifelong Learning. Brain Waves 2 (2011), February. 
[13] Slagter HA, Davidson RJ, Lutz A. Mental training as a tool in the neuroscientific study of brain and cognitive plasticity. Frontiers in Human Neuroscience 5 (2011) 1-12.

[14] Driemayer J, Boyke J, Gaser C. et al. Changes in gray matter induced by learning-revisited. Plus One 3E 2669 (2008) 1-5.

[15] Nikolaidis A, Voss MW, Lee H et al., Parietal plasticity after training with a complex videogame is associated with individual differences. Frontiers in Human Neuroscience 8 (2014) 1-11.

[16] Lent R, Tovar-Moll F. How can development and plasticity contribute to understanding evolution of the human brain? Frontiers in Human Neuroscience 9 (2015) 1-16.

[17] Ewan BM. Epigenetics and learning. Trends in Neuroscience and Education 4 (2015) 108-111.

[18] Dance A. Connectomes make the map. Nature 526 (2015) 147-149.

[19] Zatorre RJ, Fields RD, Johansen-Berg H. Plasticity in gray and white: Neuroinaging changes in brain structure during learning. Nature Neuroscience 15 (2012) 528-536.

[20] Frank C, Land WM, Popp C, Schack T. Mental representation and mental practice: Experimental investigation on the functional links between motor memory and motor imagery. Plus One 995175 (2014) 1-12.

[21] Kraeutner SN, MasKenzie LA, Westwook DA et al. Characterizing skill acquisition through motor imagery with no prior physical practice. Journal of Experimental Psychology 42 (2016) 257-265.

[22] Schack T, Essig K, Frank C, et al. Mental representation and motor imagery training. Frontiers in Human Neuroscience 8 (2014) 1-10.

[23] Lipsky RH. Epigenetic mechanisms regulating learning and long-term memory. International Journal of Developmental Neuroscience 31 (2013) 353-358.

[24] LeBeau AJ, Rissman EF. Running-induced epigenetic and gene expression changes in the adolescent brain. International Journal of Developmental Neuroscience 31 (2013) 382-390.

[25] Dieckmann P, Molin F, Lippert A, et al. The art and science of debriefing in simulation: Ideal and practice. Medical Teacher 31 (2009) e297-e294.

[26] Gerbier E, Toppino TC. The effect of distributed practice: Neuroscience, cognition and education. Trends in Neuroscience and Education 4 (2015) 49-59.

[27] Demirel D, Butler KL, Halic T, et al. A hierarchical task analysis of cricothyroidotomy procedure for a virtual airway skills trainer simulator. American Journal of Surgery 2015; Published online October 16.

[28] Bathalon S, Dorion D, Darveau S, Martin M, Cognitive skills analysis, kinesiology and mental imagery in the acquisition of surgical skills. Journal of Otolaryngology 34 (2005) 328-332.

[29] Weller JM, Nestel D, Marshall SD, et al. Simulation in clinical teaching and learning. MJA 9 (2012) 594.

[30] Rohbanfard H, Proteau L. Live vs. Video presentation techniques in the observational of motor skills. Trends in Neuroscience in Education 2 (2013) 27-32.

[31] Taylor PJ, Russ-Eft D, Chan DWL. A meta-analytic review of behaviour modeling training. Journal of Applied Psychology 90 (2005) 692-709.

[32] Frank C, Land WM, Schack T. Mental representation and learning: The influence of practice on the development of mental representation structure in complex action. Psychology of Sport and Exercise 14 (2013) 353-361.

[33] Klasen M, Weber R, Kirchner TTJ. Neural contributions to flow experience during video game playing. Scan 7 (2012) 485-495.

[34] Rodriguez P, Zhou W, Barrette DW. Multimodal randomized functional MR imaging of the effects of methylene blue in the human brain. Radiology 2016; Article in press.

[35] Bavelier D, Green CH, Pouget A, et al. Brain plasticity through the life span: Learning to learn and action video games. Annual Review of Neuroscience 35 (2012) 391-416.

[36] Iaria G, Petrides M, Dagher A, et al. Cognitive strategies dependent on the hippocampus and caudate nucleus in human navigation: Variability and change with practice. Journal of Neuroscience 23 (2003) 5945-5950. 
[37] Mohamed AD. Neuroethical issues in pharmacological cognitive enhancement. Cognitive Science 52014 533-549.

[38] Pusic MV, Kessler D, Szyld D, et al. Experience curves as an organizing framework for deliberate practice in emergency medicine learning. Academic Emergency Medicine 19 (2012) 1476-1480.

[39] Herholz SC, Zatorre RJ. Musical training as a framework for brain plascicity: Behavior, function and structure. Neuron 76 (2012) 486-502.

[40] VanDongen EV, Kersten IHP, Wagner IC, et al. Physical exercise performed four hours after learning improves memory retention and increases hippocampal pattern similarity during retrieval. Current Biology 26 (2016) 1-6.

[41] Zeidan F, Johnson SK, Diamond BJ. Mindfulness meditation improves cognition: Evidence of brief mental training. Conciousness and Cognition (2010) 1-9.

[42] Hardy JL, Nelson RA, Thomason ME, et al. Enhancing cognitive abilities with comprehensive training: A large, online, randomized, active-controlled trial. Plos One 10 (2015) 1-17.

[43] Alam F, Boet S, Piquette D, et al. E-Learning optimization: The relative and combined effects of mental practice and modeling on enhanced podcast-based learning-a randomized controlled trial.

[44] Bavelier D, Levi DM, Li RW, Dan Y, Hensh TK. Removing Brakes on Adult Brain: From molecular to Behavioral interventrions. Plasticity. J Neuroscience 4 (2010) 14964 - 14971. 